\title{
Grenzen im Spiegel des Rechts
}

\author{
Timo Tohidipur
}

\begin{abstract}
Grenzen suggerieren Stabilität und Rechtssicherheit. Sie sind das Ergebnis von Wanderungen und Eroberungen, bestenfalls das Resultat friedlicher Einigung. Doch Grenzen inkludieren und exkludieren nicht nur bezüglich eines Territoriums, sondern auch bezüglich der Rechte, die den Menschen in den jeweiligen Rechtsräumen zugestanden werden. Gleichzeitig sind Grenzen weit weniger strikt gesetzt, als es der Blick auf politische Landkarten suggeriert. Streitigkeiten über Grenzen sind stetig präsent und werden teils politisch oder kriegerisch gelöst, teils vor Gerichten verhandelt.
\end{abstract}

\section{Schlagwörter}

Grenzschutz, Rechtsgeltung, Souveränität, Staatsgrenze, Territorialität

\section{Einleitung: Grenze als Strukturprinzip des Rechts}

Grenzen spielen im Recht auf vielfältige Weise eine bedeutsame Rolle. Im Fokus sind dabei nicht nur die sichtbaren räumlichen Umgrenzungen, nicht nur die Errichtung von befestigten Grenzanlagen wie Mauern, Gräben oder Zäunen (zu deren weltweiten Renaissance vgl. Brown 2018, S. 26f.), sondern auch die unsichtbaren Grenzen auf See und in der Luft, die trotz mangelnder Visualisierung Rechtswirkung erzeugen. Eng verbunden mit dem umgrenzten Territorium ist die Frage, welche Institutionen, Organisationen, Organe und Behörden Kompetenzen haben und in welchen Grenzen sie tätig werden dürfen. Die Garantie von Grund- und Menschenrechten ist eine humanitäre Verantwortung, die nicht an Grenzen Halt machen sollte, doch sind sie als Rechtstexte in ihrer Reichweite und Wirkung oft ausdrücklich oder strukturell begrenzt. Der Mensch ist Rechtssubjekt und doch mit Blick auf seine Rechtswahrnehmung einer territorialen Entität, in unseren Zeiten einem Staat, zugeordnet, wodurch die dem Menschen mögliche Einforderung von Rechten durch seine Staatsbürgerschaft ausgestaltet und zugleich begrenzt ist. Grenzen definieren so auch soziale Verhältnisse (vgl. Hess/Karakayali 2017, S. 27).

Während kontinuierlich Grenzkonflikte zwischen Staaten oder innerhalb von Staaten, teils friedlich, teils durch Gewalt und Krieg, ausgefochten wurden und werden (vgl. Jennings 1963, S. 1ff., 79ff.), standen in der Zeit nach dem Zweiten Weltkrieg eher die positiven und konstruktiven Ideen überstaatlicher Kooperation im Sinne einer Globalisierung samt der immer weiter fortschreitenden Herausarbeitung und Durchsetzung (menschen)rechtlicher Garantien weltweit im Fokus. Auf der Basis einer an Gewaltfreiheit orientierten Weltordnung der Vereinten Nationen offerierte sich die friedliche Streitbeilegung als Leitmotiv, während zugleich eine internationale Weltwirtschaftsordnung (Welthandelsorganisation, WTO etc.) etabliert wurde, die Handelskonflikte zu verhindern und gemeinsame, grenzüberschreitende Regeln für den internationalen Handel zu etablieren suchte. Das supranationale Kooperationsprojekt Europäische Union (EU) sollte die (national)staatlichen Egoismen überwinden und wurde damit direkt oder indirekt Vorbild für entsprechende Tendenzen in Südamerika (Mercosur, Andengemeinschaft), Osteuropa/Nordasien (Eurasische Wirtschaftsunion, EAWU) oder Westafrika (Westafrikanische Wirtschaftsgemeinschaft, ECOWAS). Dabei geriet der (National-)Staat nie 
gänzlich aus dem Blick, behielt seine Rolle als Organisator gesellschaftlicher Entwicklung, insbesondere mit Blick auf soziale Sicherung und Arbeitsorganisation, und blieb als vermeintlich sicherer Rückzugsort in Zeiten der Krise oder als letztverbindlicher Garant von Sicherheit und Recht in seinen Grenzen bestehen. Die zeitgenössische Auseinandersetzung mit Grenzen und Recht findet daher einerseits in Abhandlungen zum Staatsrecht und der Staatslehre unter dem Stichwort „Staatsgebiet“ statt (Vitzhum 2004, S. 163; Isensee 2018, S. 77; Schöbener/Knauff 2019, S. 3, 88ff.), andererseits findet zugleich die überstaatliche Perspektive ausführliche Behandlung im Völkerrecht unter den Stichworten „Raum“, „Gebiet“, „Territorium“ und „Souveränitätsbereich“ (Verdross/Simma 1984, S. 599; Ipsen 2019, S. 77; Proelß 2019, S. 463). Neben unterschiedlichen Grenzdimensionen und den historisch-funktionalen Aspekten von Grenzen fokussieren die folgenden Ausführungen die Bedeutung von Grenzen zwischen Staaten, der Entstehung und Neuordnung von Staatsgrenzen und deren Auswirkung auf Identitätsbestimmung und Partizipation sowie einen kurzen Blick auf Debatten um Grenzen im Kontext der internationalen Wirtschaftsordnung und der Sicherheitsdebatte. Kontrastierend dazu ist schlaglichtartig die überstaatliche Zusammenarbeit in den Blick zu nehmen, ebenso wie ein kritischer Blick auf Grenzen.

\section{Bedeutung, Funktion, Geltung von Grenzen im Recht}

\subsection{Grenzdimensionen}

Wenn man im Recht von Grenzen spricht, dann sind zunächst Geltungsgrenzen des Rechts gemeint. Diese können zunächst ganz unmittelbar auf staatliche Grenzen bezogen werden, die die Grenzen der Geltung des staatlich gesetzten Rechts grundsätzlich markieren. Überdies sind Geltungsgrenzen auch auf überstaatliche, transnationale oder supranationale Zusammenschlüsse wie die EU oder ECOWAS bezogen, wo der gemeinsame Rechtskodex über Staatsgrenzen hinweg Geltung haben kann. Das internationale Recht bzw. Völkerrecht kann grundsätzlich weltweit Rechtswirkung beanspruchen, doch im Einzelfall bestehen Wirkungsgrenzen, deren konkrete Reichweite je nach Rechtsnorm oder staatlicher Umsetzung variiert. Jenseits dieser öffentlich-rechtlich begründeten Rechtsgeltung können die Geltungsgrenzen des Rechts auch privatrechtlich, also mit Blick auf Privateigentum, verstanden werden. Aus deutscher Sicht berechtigt das private Eigentumsrecht einer Sache dazu, andere von der Nutzung einer Sache auszuschließen ( $\$ 903$ Bürgerliches Gesetzbuch, BGB). Zugleich ist das Eigentum eines Grundstücks seinerseits begrenzt und erstreckt sich „auf den Raum über der Oberfläche und auf den Erdkörper unter der Erdoberfläche“, und zwar in solcher Höhe und Tiefe, soweit es für eine*n Eigentümer* in von Interesse sein kann ( $\$ 905$ BGB). Doch darf der Zugriff auf das Grundwasser zum Beispiel dann einer gesonderten staatlichen (wasserrechtlichen) Regelung unterworfen werden, soweit dies zur Sicherung einer funktionsfähigen öffentlichen Wasserversorgung erforderlich ist (Bundesverfassungsgericht, amtliche Sammlung, BVerfGE 58, 300, 332).

Vertikal nach unten, unterhalb des Staatsgebiets, wirkt das staatliche Recht unbegrenzt, also letztlich bis zum Erdmittelpunkt (vgl. Khan 2019; vgl. bildlich Ipsen 2018, S. 80). In ähnlicher Weise ist der Himmel über einem Privatgrundstück nicht grenzenlos im privaten Eigentum, denn ansonsten wäre jeder Flugverkehr von Zustimmungen aller Privateigentümer*innen unterhalb der Flugroute abhängig. Hier greift wieder das staatliche Recht, jedenfalls bis zu 
einer Höhe von ca. 100 Kilometern, also die Grenze zwischen Luftraum und Weltraum (die sogenannte Kármán-Linie). Der Weltraum unterliegt seinerseits gemäß Art. II WRV (Vertrag über die Grundsätze zur Regelung der Tätigkeiten von Staaten bei der Erforschung und Nutzung des Weltraums einschließlich des Mondes und anderer Himmelskörper, BGBl. 1969, II, S. 1969) keiner nationalen Aneignung, wodurch dort auch keine Hoheitsgewalt beansprucht werden kann. Der Weltraum ist hoheitsfreier Gemeinschaftsraum (vgl. Wolter 2002, S. 941). Die Weltraumfahrt organisiert sich grundsätzlich über Staatsgrenzen hinweg in überstaatlicher Kooperation. Horizontal wird ein Staatsgebiet durch die Staatsgrenzen zu anderen Staaten oder nicht staatlich zugewiesenem Territorium, wie z.B. die Hohe See, deren Nutzung internationalem Recht unterliegt, begrenzt. Mithin bleibt der Staat trotz europäischer und internationaler Kooperationszusammenhänge innerhalb der internationalen Gemeinschaft die zuvorderst rechtlich relevante Organisationsform von Gesellschaften, dessen zentrale Stellung ihn als Hauptakteur für die relative Sicherung eines Gemeinwohls und zugleich die Durchsetzung privater Rechte auszeichnet (vgl. Vitzthum 2004, S. 165; Tohidipur 2015, S. 109). Daher werden die folgenden Ausführungen zu Grenze und Recht vom Staat ausgehen.

\subsection{Historisch-funktionale Annäherung an Grenzen im Recht}

Grenzen im rechtlichen und damit letztlich auch im politischen Sinne bezeichnen den Umfang eines Teils der Erdoberfläche, eines bestimmten Territoriums. Dies lässt sich für das Wort Grenze schon begrifflich ableiten: Grundlage ist das altslavische Wort granica (polnisch, bulgarisch, bosnisch) bzw. graniza (russisch, bulgarisch), das also (Beginn und) Ende eines Raumes bezeichnet (vgl. Schmieder in diesem Band). Dieser Raum, der begrenzt wird, ist zunächst als geografischer Raum zu verstehen. Doch rechtlich geht es eben nicht nur um das Territorium oder den geografischen Raum an sich, sondern um die Frage, welche (Rechts-)Regeln dort gelten.

Aus der Sicht sesshafter Lebensgemeinschaften geht es um die Abgrenzung eines Gebietes wie z.B. von Wohnflächen und landwirtschaftlich genutzten Flächen für die eigene Familie oder Gruppe (vgl. Bredow 2014, S. 17). Es werden Ressourcen eines bestimmten Raumes exklusiv einer bestimmten Gruppe von Menschen zugeschrieben, wodurch eine raumbezogene Identifikation erwächst, die zugleich exkludierend auf raumfremde Konkurrenten wirkt und damit auch erhebliches Konfliktpotenzial birgt (vgl. Khan 2019). Wenn davon auszugehen ist, dass jedes Leben auf der Erde Platz benötigt, ergibt sich daraus die Frage, wem welcher und wie viel Platz zusteht (zur verbundenen Frage der Geopolitik vgl. Bredow 2014, S. 18ff.). In diesem Zusammenhang werden Machtpositionen generiert, die über das Private hinaus auch Herrschaft mit Bezug zu einem konkreten Gebiet begründen können. Dann geht es nicht mehr nur um die eigene Nutzung, sondern um die politisch verstandene Ausübung von Herrschaftsgewalt über ein bestimmtes Gebiet. Damit konstituieren Grenzziehungen zugleich Geltungsgrenzen von Recht und Macht. Diese Organisation des Raums hat mithin auch friedensstiftende Wirkung (umfriedetes Gebiet), denn sie weist Zugehörigkeits- und Nutzungsrechte ausdrücklich zu und erzeugt so Rechtssicherheit und Transparenz. Zur Begründung bestimmter Grenzziehungen sind die Legitimations- und Legalisierungsmechanismen bedeutsam, die die Grundlage der rechtlichen Zuschreibung bilden. Dies kann durch Berufung auf göttliche Legitimation erfol- 
gen, so z.B. durch Bezugnahme auf den römischen Grenzgott Terminus (vgl. Khan 2019), oder ganz unmittelbar auf konsensual festgelegten Verfahren beruhen.

\subsection{Territorium der Rechtsgeltung: Konstituierung von Staatsgrenzen (Land, Wasser, Luft)}

Die für die Staatspraxis immer noch relevante Drei-Elemente-Lehre von Georg Jellinek sieht im Staat die „mit ursprünglicher Herrschermacht ausgerüstete Körperschaft eines sesshaften Volkes“ (Jellinek 1960 S. 183), formuliert somit als konstituierende Merkmale des Staates eine Staatsgewalt, ein Staatsvolk und eben ein Staatsgebiet, als ein abgegrenzter Teil der Erdoberfläche, der als ausschließlicher Herrschaftsbereich verstanden wird (vgl. Schöbener/Knauff 2019, S. 89). Ausgangspunkt der zeitgenössischen Überlegung, wie Grenzen gezogen werden, ist die Souveränität der Staaten, zurückgehend auf den Westfälischen Frieden 1648, der das Staatssystem nicht auf Grundlage von Macht, sondern auf der Basis rechtlicher Gleichordnung festschrieb: Der souveräne Staat soll seine Grenzen und damit sein Territorium und den Zugang zu selbigem festlegen können (vgl. Khan 2012, S. 233; Känner 2019, S. 18f.; siehe auch Herrmann/Vasilache in diesem Band). Hier hat der Staat die alleinige Kompetenz (vgl. Vitzthum 2004, S. 168; Ipsen 2018, S. 79), hier wird also das staatliche Territorium mit der staatlichen Souveränität verknüpft, die den Staat die „unabhängige, oberste Regelungsmacht“ zuweist (Schliesky 2004, S. 25; vgl. auch Khan 2004, S. 2; Crawford 2019, S. 192). Diese Souveränität umfasst zugleich eine innere Souveränität, also Verfassungsautonomie und damit die Fähigkeit, eine Ordnung auf dem Staatsgebiet zu organisieren, wie auch die äußere Souveränität, also das unabhängige und selbständige Handeln nach Maßgabe des Völkerrechts, ohne einer anderen Autorität unterworfen zu sein (vgl. Schorkopf 2017, S. 2; Ipsen 2019, S. 139f.). Den Zusammenhang zwischen Territorium, Staatsgrenze und Souveränität betont auch der Internationale Gerichtshof (IGH) in einer frühen Entscheidung aus dem Jahre 1978 (IGH, Agean Sea Continental Shelf Case [Greece vs. Turkey], Urteil v. 19.12.1978, insb. Abs. 84). Die Definition eines Territoriums durch Abgrenzung von anderen Territorien (vgl. zum Konzept des Territoriums im Recht: Crawford 2019, S. 191ff.), also durch Grenzsetzung, ist Grundbedingung für die Möglichkeit der Ausübung territorialer Souveränität, die von einer klaren, gleichsam absoluten Trennung von Hoheitssphären benachbarter Staaten ausgeht (vgl. Khan 2019). Die hierfür maßgebliche Grenzlinie ist nur im horizontalen Verständnis eine Linie, denn wenn die vertikale Achse mitgedacht wird, also die Souveränität über Luft oberhalb und Boden unterhalb der Oberfläche, dann bildet die Grenze ihrerseits vertikal eine Fläche. Dies hat ganz praktische rechtliche Auswirkungen: So ist es einem Staat beispielsweise nicht gestattet, unter dem horizontalen Grenzverlauf hindurch im tiefen Boden des Nachbarstaates nach Bodenschätzen zu bohren oder den Luftraum über dem Territorium ohne ausdrückliche Genehmigung zu nutzen. Bei den Seegrenzen besteht gemäß dem Seerechtsübereinkommen der Vereinen Nationen (SRÜ) ein abgestuftes System. So stehen jedem Küstenstaat ab der Küste zwölf Seemeilen als Hoheitsgewässer (Küstenmeer, vgl. Art. 2ff. SRÜ) zu, die die wirtschaftliche Nutzung einschließlich des Rohstoffabbaus garantiert und zugleich grundsätzlich ein (friedliches) Durchfahrtsrecht für zivile Schiffe anderer Staaten vorsieht (Art. 17ff. SRÜ). Schwieriger werden die Berechnungen, wenn Küstenstaaten auch vorgelagerte Inseln haben, was bezüglich der eigenen Hoheits- bzw. Nutzungsrechte leicht zu Konflikten zwischen Staaten führen kann (vgl. Arnauld 2019, S. 382; siehe nur den andauernden Streit zwischen Griechenland und der Türkei im Mittelmeer). 
An die Hoheitsgewässer schließt eine weitere zwölf Seemeilen umfassende Anschlusszone an (Art. 33 SRÜ), die als Sicherheitszone polizeilich und zollrechtlich überwacht wird. Vor allem aber beginnt dort ebenfalls die Ausschließliche Wirtschaftszone (AWZ, vgl. Art. $55 \mathrm{ff}$. SRÜ), die auf und unterhalb des Wassers (der sogenannte Festlandsockel, vgl. Art. 76 SRÜ) alleinige wirtschaftliche Nutzung durch den Küstenstaat vorsieht, also die Erforschung, Ausbeutung, Erhaltung und Bewirtschaftung der lebenden und nicht lebenden Ressourcen, und sich dabei über grundsätzlich 200 Seemeilen erstreckt. Die AWZ umfasst nicht den Luftraum, worin ein deutlicher Unterschied zum staatlichen Territorium zu erblicken ist. Zudem werden allgemeine Rechte dritter Staaten garantiert, wie z.B. freie Schifffahrt, freier Überflug und freie Verlegung unterseeischer Kabel und Rohrleitungen (vgl. Proelß 2019, S. 505). Danach beginnt die Hohe See, die keine territoriale Zuordnung kennt und als gemeinsames Erbe der Menschheit verstanden bzw. als globaler Staatengemeinschaftsraum bezeichnet wird und wie der Weltraum allen (Staaten) offen steht, wobei auf eine faire und angemessene Nutzung, d.h. zum Wohle der gesamten Menschheit und im Sinne gerechter Verteilung unter Einbeziehung benachteiligter Staaten, geachtet werden soll (Feichtner 2019, S. 10; Proelß 2019, S. 514f.; vgl. auch Art. 87 SRÜ). Die Hohe See unterliegt damit grundsätzlich keinem staatlichen Recht, ist aber auch kein rechtloser Raum, da es beispielsweise eine Pflicht zur Hilfeleistung bei Gefährdungen für Menschen gemäß Art. 98 SRÜ gibt.

Der völkerrechtliche Grundsatz der Achtung der territorialen Integrität der Staaten verbietet zunächst grundsätzlich alle Staatshandlungen im Ausland, durch die in die Gebietshoheit des Territorialstaates eingegriffen wird (vgl. Verdross/Simma 1984, S. 276). Diese Gebietshoheit kann allerdings durch Einwilligung partiell aufgehoben oder für einen auswärtigen Staat geöffnet werden, so z.B. für die Errichtung einer Militärbasis oder auch nur für besondere Überflugrechte. Das Handeln des auswärtigen Staates ist auf die Reichweite der Genehmigung begrenzt. Eine Gewährung von (vertraglichen) Rechten darf auch nicht durch Gewalt oder Drohung erzwungen werden (Art. 52 Wiener Übereinkommen über das Recht der Verträge, WVK), wobei nicht eindeutig geklärt ist, ob jenseits der Androhung militärischer Gewalt auch die Androhung negativer wirtschaftlicher Konsequenzen schon als Drohung in diesem Sinne verstanden werden kann (vgl. Ipsen 2019, S. 512f.). Die Öffnung des eigenen Staatsgebietes für Beamt*innen oder Soldat*innen eines anderen Staates entbindet jedoch den gewährenden Staat nicht von der Verantwortung für die rechtskonforme Nutzung durch den auswärtigen Staat (vgl. Verdross/Simma 1984, S. 640). So hat Deutschland dafür Sorge zu tragen, dass vom USStützpunkt in Rammstein aus keine völkerrechtswidrigen Drohneneinsätze geflogen werden (OVG Urt. v. 19.3.2019, Az. 4 A 1361/15). Grenzen konturieren also auch ein Territorium der Pflichten für den Staat (vgl. hierzu schon Jellinek 1960, S. 367).

Die Verknüpfung von umgrenztem Territorium und Souveränität ist indes nicht zwingend, wie sich gegenwärtig am Beispiel Syriens oder Libyens zeigt, wo anerkannte Grenzen nicht mehr mit einer territorialen Souveränität im Inneren übereinstimmen. Umgekehrt muss die innere Kohärenz einer politischen Gemeinschaft auf einem bestimmten Territorium nicht unbedingt die Existenz eines Staates mit entsprechenden Grenzen bedeuten, wie die komplizierte Debatte um die Staatlichkeit Palästinas zeigt, zumal die Geltendmachung von Rechten in der Internationalen Gemeinschaft oder der Teilnahme an völkerrechtlichen Gerichtsverfahren grundsätzlich nur Staaten gewährt wird (vgl. Özdemir 2020, S. 1). 


\section{4 Änderung von Grenzverläufen: Übertragung, Zerfall, Neuordnung, Zession, Annexion}

Die einmal gesetzte Grenze und das damit definierte Territorium sind nicht unabänderlich. Die konkrete Grenzziehung erfolgt im Wege eines völkerrechtlichen Grenzvertrages, setzt grundsätzlich Konsens der Beteiligten und die gegenseitige, von Gewalt oder Gewaltandrohung befreite Anerkennung als gleichwertige Vertragspartner voraus (vgl. Verdross/Simma 1984, S. 274, 610). Denkbar, aber praktisch kaum mehr relevant ist die Möglichkeit, neues, ,staatsfreies', also nicht einem Staat zugeordnetes oder von einem Staat aufgegebenes Gebiet (im Völkerrecht als res nullius bezeichnet) dem eigenen Staatsgebiet hinzuzufügen (vgl. dazu Vitzthum 2004, S. 170; Crawford 2019, S. 191). Nicht selten waren und sind hingegen staatliche Neuordnungen zu organisieren, selbst in den letzten Jahrzehnten, wie z.B. im Nachgang der Auflösung des russischen Kaiserreichs 1917, des Zerfalls des Osmanischen Reichs 1918/1920, der Dekolonisierung in Asien und Afrika nach dem Zweiten Weltkrieg und des Zusammengehens zweier deutscher Staaten 1990/91. Wenn der Vorgängerstaat vollständig untergeht, geht das Völkerrecht von Zergliederung oder Dismembration (dismemberment) aus, also dem Zerfall eines Staates in mehrere souveräne Staaten, wie z.B. im Fall der UdSSR (1991) und der Tschechoslowakei (1992), mit der Folge, dass auch die Grenzen neu geordnet werden müssen. Der Fall Jugoslawiens liegt rechtlich undeutlicher, da sich zunächst Slowenien und Kroatien, sodann Mazedonien losgesagt hatten, bevor sich ein weiteres Auseinanderbrechen vollzog, wodurch die Nachfolgestaaten rechtlich auf gegenseitige und externe Anerkennung zur Sicherung der eigenen territorial-staatlichen Existenz angewiesen waren (vgl. Arnauld 2019, S. 46).

Eine Neuordnung kann zudem als Fusion erfolgen, d.h. als Zusammenschluss mehrerer Staaten zu einem Staat, wie im Beispielfall von Tanganjika und Sansibar, die sich 1964 zu Tansania zusammenfanden. Zu unterscheiden hiervon ist die Inkorporation, also der Beitritt eines Staates zu einem anderen, wie es im Fall der Wiederherstellung der deutschen Einheit sichtbar wurde (vgl. ebd.). Durch Zession kann die territoriale Souveränität über ein bestimmtes Gebiet von einem Staat auf einen anderen übertragen oder ganz aufgegeben werden (vgl. Klein 1980, S. 185). Einer Zustimmung durch Dritte (Staaten) bedarf es grundsätzlich nicht, da durch die vertragliche Übertragung auch kein neuer Staat entsteht. Anwendungsfall derartiger Zession oder Abtretung ist zumeist die Neuregelung von Grenz- und Gebietsfragen in Friedensverträgen (vgl. Vitzthum 2004, S. 170). Zuletzt ist auch die Sezession, also die Abspaltung oder Abtrennung eines Gebietsteils von einem Staat, möglich und wird nicht selten auf das Selbstbestimmungsrecht der Völker gestützt. Unter diese Kategorie fällt etwa die Sezession Bangladeschs von Pakistan 1971 und zuletzt die Abspaltung des Süd-Sudan vom (Nord-)Sudan, was letztlich auf die Dekolonisierung 1956 zurückgeht (vgl. Arnauld 2019, S. 46; Brunner 2013, S. 38). Die Idee des Selbstbestimmungsrechts der Völker ist nicht einfach zu handhaben, wenn sie über ihren Ursprung in der rechtebasierten Dekolonisierung hinaus reklamiert wird (vgl. Arnauld 2019, S. 26f.). So ist schon die Definition des sich selbst bestimmenden Volkes schwierig, zumal wenn sich nur Teile einer Bevölkerung eines Staates separieren möchten. Darüber hinaus ist die Reichweite der Selbstbestimmung nicht deutlich konturiert. Keine zulässige Form der Neuordnung stellt der zwangsweise bzw. auf Druck oder durch Gewalt erfolgte Zusammenschluss dar (vgl. Ipsen 2019, S. 512). Vielmehr sieht das Völkerrecht hierin eine Annexion (vgl. Arnauld 2019, S. 27, 498 zur Krim). 
All dies sind politisch-rechtliche Prozesse der Neuordnung von Grenzen, bei denen es stets um staatliche Macht- und Wirtschaftsinteressen wie etwa den Zugang zu Rohstoffen oder die territoriale Kontrolle taktisch (militärisch) wichtiger Räume geht. Als Begründungsreservoir dienen zudem auch sehr häufig historisch-ethnische Argumentationslinien, die inkludierende und exkludierende Effekte statuieren.

\subsection{Inklusion und Exklusion: Aspekte räumlicher Identitätsbestimmung und Partizipation durch Recht}

Rechtlich gesetzte Grenzen eines politischen Gemeinwesens - eines Staates - unterscheiden zwischen innen, als der Sphäre des Zugehörigen, und außen, als der Sphäre des Fremden (vgl. Schorkopf 2017, S. 47), definieren in der Folge die einen Menschen als Mitglieder und die anderen als Fremde (vgl. Benhabib 2008, S. 13). An dieser Bestimmung von Zugehörigkeit knüpfen auch etwaige Rechte auf Partizipation und Teilhabe an. Historisch symbolisierten Grenzen oft kulturelle oder zivilisatorische Trennlinien, die auf der einen Seite die (eigene) Zivilisation und auf der anderen die (fremde) ,Barbarei‘ zu erblicken glaubten (vgl. Bredow 2014, S. 36f.). Hier bricht sich im historischen Rückgriff auf das 18. und 19. Jahrhundert ein letztlich durch die dunkle Seite der Aufklärung begünstigter Rassismus bahn, der in einem vermeintlich rationalen Zugriff äußere Merkmale (z.B. Hautfarbe) und innere (z.B. Moral, Kultur) mit Orten auf der Welt verknüpft (hier insbesondere Asien und Afrika) und so in Abgrenzung zu diesen Gesellschaften eine Rangordnung konstruiert, die die weißen Europäer*innen an der Spitze sieht (vgl. Scheffer 2018, S. 37). Diese diskriminierende Sicht, die über die Darstellung von unterschiedlichen Zugehörigkeiten zu Rechtsräumen hinaus auch Hierarchien der Wertigkeit zu konstruieren versucht, ist selbst heute leider noch zuweilen deutlich präsent.

Die in der deutschen Staatslehre verwendete Bezeichnung Staatsvolk zielt auf einen auf Dauer angelegten Verbund von Menschen ab, über den ein Staat die Hoheitsgewalt ausübt (im Sinne der Gebietshoheit), während der Staat im Fall des Aufenthaltes außerhalb der Staatsgrenzen die Personalhoheit innehat (vgl. Ipsen 2019, S. 122). In Anschluss an Georg Jellinek (1960, S. 406) bilden „die dem Staate zugehörigen Menschen [...] in ihrer Gesamtheit das Staatsvolk“. Diese Zugehörigkeit wird im ersten Zugriff juristisch über die Staatsangehörigkeit bestimmt, also ein (staatliches) Dokument, das die Zugehörigkeit individuell und für den Rechtsverkehr verbindlich dokumentiert. Orientierungsgrundlage ist einerseits die geografische Zufälligkeit des Geburtsortes, sodann die Frage der Abstammung. Die Idee des Staatsvolkes knüpft indes, basierend auf einer europäischen Denktradition, auch an andere Aspekte an: Bei der Beschreibung des neuzeitlichen ,modernen`Staates verbindet sich die Idee der Souveränität, in der Form der Volkssouveränität, mit der Idee der Nation, denn das Volk als originärer Träger der Herrschaftsgewalt sei beauftragt, die auf historischen Wurzeln basierende und alle Teile des Volkes verbindende Einheit, mithin als nationale Einheit zu konstituieren und damit die im Nationalstaat zusammengefasste Nation zu legitimieren (vgl. Schliesky 2004, S. 23; weiterführend: Habermas 2015, S. 146ff.). Die hierbei vorausgesetzte, historisch-kulturell homogene Gemeinschaft ist indes, sowohl auf Deutschland als auch auf die EU bzw. Europa bezogen, eine - zuweilen stark romantisierende - Imagination, eine Projektionsfläche für unterschiedliche Desiderate wie Gefühle von Sicherheit oder Zusammengehörigkeit und damit für eine geradezu „unwirkliche politische Einheit“ (Schulze 1990, S. 16f.), die nicht selten in 
Kriegs- oder Krisenzeiten formuliert wird, um politisch zu mobilisieren (vgl. Guérot 2019, S. 35). Ein Staat kann grundsätzlich innerhalb seiner Grenzen bestimmen, wer den Nachweis der Zugehörigkeit erhält (Staatsangehörigkeit), und kann zugleich grundsätzlich regeln, welche Rechte Menschen mit anderen Aufenthaltsrechten erhalten (Dauer des Aufenthalts, Zugang zur Gesundheitsversorgung oder sozialen Sicherung etc.) und letztlich auch ganz grundlegend, wer die Grenze überhaupt übertreten darf. Der Staat hat im Rahmen seiner Verfassung hier Gestaltungsfreiheit, was dann problematisch wird, wenn Minderheiten von der Aussicht auf staatsbürgerliche Gleichbehandlung mittelbar oder unmittelbar ausgegrenzt werden, wie z.B. anhand der Abstammung und/oder der religiösen Zugehörigkeit (vgl. den Streit um das geänderte Staatsbürgerschaftsrecht in Assam/Indien, dazu: Shah/Tripathi 2020). Der Entzug der Staatsangehörigkeit führt letztlich zur Staatenlosigkeit und damit gewissermaßen zur Rechtlosigkeit des Individuums, da die Durchsetzung von Rechten leider durchweg staatszentriert erfolgt. Gerade in diesem Zusammenhang hatte schon Hannah Arendt (1949/2011, S. 401) für das Recht Rechte zu haben, als existenzielles, mithin einziges echtes Menschenrecht, plädiert. Gleichwohl sind diese staatlich bedingten Rechte bei Mitgliedschaft in einer überstaatlichen Organisation (z.B. EU) durch ihr überstaatliches Recht determiniert, wodurch eine staatliche Willkür verhindert werden soll. Die EU modifiziert die Zuordnung durch die Unionsbürgerschaft, die etwa Bürger*innen aus anderen EU-Mitgliedstaaten kommunales Wahlrecht in jeweils anderen EU-Mitgliedstaaten einräumt (vgl. Art. 22 AEUV; zur politischen und sozialen Inklusion vgl. Tohidipur 2011). Aus demokratischer Perspektive erfordert die Selbstbestimmung einer Gruppe eben gerade die Bestimmbarkeit dieses Kollektivs, verstehbar als die personelle Grenze des Demos (vgl. Schmalz 2013, S. 179). Eine etablierte staatsbürgerliche Solidarität kann sich in dem Maße über die Grenzen des Nationalstaates hinaus erweitern, in dem die Bürger*innen von supranationalen Entscheidungen nicht nur betroffen, sondern daran nach demokratischen Verfahren auch beteiligt werden (vgl. Habermas 2015, S. 151). Zumal auf einer darunter liegenden Ebene, z.B. bei föderal organisierten Staaten, die Grenzen der territorialen Untergliederungen, wie die der Bundesländer in Deutschland, ebenfalls lokale und regionale Entwicklungen widerspiegeln und darüber hinaus sogar städtische Bürgerschaften zusätzlich zur staatlichen diskutiert werden (vgl. Bauböck 2020), während zugleich eine gesamtbundesstaatliche Zuordnung bestehen bleibt (vgl. Khan 2004, S. 33).

\subsection{Staatsgrenze und Sicherheitsdiskurs: Wirkungen von Außen- und Binnengrenzen}

Während Grenzen für den Einzelnen gleichbedeutend mit einem Schutzraum sein können, zeigen sich aus der Sicht der Staaten mit Blick auf die Kontrolle von Grenzen stets Bezüge zu Sicherheit und Überwachung, so zur Bekämpfung von organisierter Kriminalität bis hin zum Schutz der Integrität des Staatsgebietes, dem Schutz der einheimischen Wirtschaft, dem Schutz der Gesundheit der eigenen Bevölkerung oder auch der Verhinderung von Migration bzw. Flucht über die Grenze, was in der Konsequenz zur Versicherheitlichung (securitization), also zur Aufrüstung bzw. Militarisierung der Grenzen führt. Die Errichtung von Mauern und Zäunen ist dabei nur ein Modus der Grenzsicherung, der auch aktuell in vielen Regionen der Welt eine weiterhin bedeutende Rolle spielt, wie die Beispiele Indien, Saudi-Arabien, China, USA und Ungarn zeigen (vgl. Vallet/David 2017, S. 143; Brown 2018, S. 26). Die Folgen der Globalisierung werden nicht nur als Möglichkeit weltweiter Vernetzung und Kooperation, sondern auch als Grundlage globaler Unsicherheit wahrgenommen und führen so zum Rück- 
zug auf zuvor etablierte staatliche Sicherheitsvorstellungen, sicherlich auch verstärkt durch Entwicklungen nach dem Anschlag auf das World Trade Center am 11. September 2001 (Vallet/David 2017, S. 144). Die weltweite Schließung der Staatsgrenzen und die entsprechenden Einreiseverbote im Kontext der Corona-Krise 2020 verdeutlichen diese Tendenz. Weiterhin werden von Staaten des Nordens auch die weltweiten Migrationsbewegungen als Bedrohung des Lebensstandards wahrgenommen (vgl. Schaffner 2011, S. 275), sodass neben Drogenund Waffenschmuggel auch der sogenannte illegale Grenzübertritt an sich, mithin letztlich grundsätzlich jede Fluchtbewegung und deren Unterstützung, unter den Begriff „Kriminalität“ gefasst wird (vgl. z.B. Erwägungsgründe 18 und 19 der Verordnung (EU) 2016/1624 des Europäischen Parlaments und des Rates vom 14. September 2016 über die Europäische Grenzund Küstenwache, Frontex-VO). Dies ist mit Blick auf menschenrechtliche Garantien und das Flüchtlingsrecht äußerst problematisch, denn so wird jedes Flüchtlingsboot in erster Linie zum potenziellen Tatort einer Straftat wie Menschenschmuggel als Handlung organisierter Kriminalität (vgl. Tohidipur 2019, S. 251). Dabei endet der staatliche Grenzschutz nicht an der eigenen Außengrenze: So ist z.B. die Entsendung von Grenzschutzbeamt*innen in andere Staaten, insbesondere an Flughäfen oder andere verkehrsrelevante Orte, gängig, da so bereits im Vorfeld Entscheidungen hinsichtlich geplanter Grenzübertritte getroffen werden können. Der Zutritt zum staatlichen Territorium und damit das Überschreiten der Grenze, ist rechtlichen Regeln unterworfen und bedarf grundsätzlich ausdrücklicher Erlaubnis (Aufenthaltstitel/Visum, vgl. für Deutschland $\mathbb{S} 4$ AufenthG), die zumeist an konkreten Zwecken orientiert ist und stets unter dem Vorbehalt der Sicherheit steht, d.h. die einreisende oder in Deutschland bleibende Person darf keine Gefahr für die öffentliche Sicherheit und Ordnung darstellen (vgl. für die Niederlassungserlaubnis z.B. $\$ 9$ Abs. 2 Nr. 4 AufenthG). Ohne Erlaubnis liegt ein Rechtsverstoß vor, der vom Staat sanktioniert werden kann. Ausgenommen hiervon sind Menschen auf der Flucht, die um Asyl bzw. internationalen Schutz bitten, soweit sich der Staat an die Vorgaben der Genfer Flüchtlingskonvention (GFK) und vergleichbare staatliche oder überstaatliche Rechtsgarantien (z.B. Europarecht) hält. Wird der Verstoß des unerlaubten Grenzübertritts von ausländischem Militär begangen, so kann darin ein feindlicher Akt gesehen werden, der ein Recht auf Selbstverteidigung des Staates auslöst (vgl. Kapitel VII der UN-Charta). Gerade mit Blick auf die Sicherheit kommt es zu unterschiedlichen Grenzdiskursen, die eine Unterscheidung von Außen- und Binnengrenzen notwendig macht (vgl. dazu Eigmüller in diesem Band). Einmal betrifft der Grenzdiskurs die Außengrenzen von Staaten und ihre Zugangsregeln. Binnengrenzen hingegen sind Grenzen, die grundsätzlich innerhalb eines begrenzten (übergeordneten) Territoriums verlaufen und ein Teilgebiet umgrenzen. So bestehen Binnengrenzen in Deutschland zunächst zwischen den 16 Bundesländern, spielen aber im täglichen Leben keine hervorgehobene Rolle. Ausnahme sind die 2020 erfolgten Beschränkungen der Freizügigkeit im Zuge der Corona-Pandemie: Hierbei wurde auf Basis des Infektionsschutzes die Grenze zwischen den Bundesländern plötzlich zum Ort der Selektion. Auch auf überstaatlicher EU-Ebene finden sich indes Binnengrenzen, womit der damit etablierten Freizügigkeit ein Sicherheitsvorbehalt entgegensteht. Entsprechend dem Schengener Grenzkodex (SGK) dürfen innerhalb der EU Binnengrenzen grundsätzlich unabhängig von der Staatsangehörigkeit der betreffenden Personen ohne Personenkontrollen überschritten werden (Art. 22 SGK). Wenn jedoch die innere Sicherheit eines Mitgliedstaates „ernsthaft bedroht“ ist, dürfen auch die Grenzkontrollen an den EU-Binnengrenzen wieder eingeführt werden (Art. 25ff. SGK). Dies war auch zuvor schon oft genutzte Praxis, ursprünglich nach den Terroranschlägen in Europa, sodann insbesondere mit in Bezug auf Migration bzw. Flucht nach Europa seit 2015 (vgl. 
Europäischen Kommission 2015; Genova 2017, S. 11). Der EU-Gerichtshof (EuGH) hat hierzu aber deutlich gemacht, dass die Wiedereinführung von Binnengrenzkontrollen die Grenzen zwischen EU-Mitgliedstaaten nicht als Außengrenze wirken lassen dürfen, d.h. es darf keine vereinfachten Inhaftierungen, Zurückweisungen oder Abschiebungen nach Aufgriff an einer Binnengrenze erfolgen (vgl. genauer Hruschka 2019).

\subsection{Grenzen als Marktgrenzen}

Während sich Grenzen im Recht primär auf die Staatsgrenzen bzw. die Reichweite der Staatsgewalt beziehen, sind andere Bereiche menschlichen Wirkens wie Wissenschaft, Kultur, Religion, Sport, Technik oder Wirtschaft grundsätzlich nicht an ebendiese Grenzen gebunden. Vielmehr kann es durchaus im Interesse zum Beispiel eines Wirtschaftsunternehmens liegen, Verträge zum Handel oder Abbau von Rohstoffen mit einem beliebigen, also auch auswärtigen Staat oder einem in einem anderen Staat agierenden Unternehmen abzuschliessen. Dies war auch schon gängige Praxis von der Antike über das Mittelalter bis in die heutige Zeit, also auch lange vor den zeitgenössischen Formen der Globalisierung des Marktes (vgl. die Schilderungen von Triepel 1939/40, S. 1ff.; Heintzen 1988, S. 26ff.), die letztlich durch dieselbe nur ausgedehnt und intensiviert wurde. Grenzen zwischen Staaten haben so immer eine Doppelfunktion: Zum einen sorgen sie für die jeweils eine und andere Seite für Sicherheit und Sicherung, andererseits sind sie der Ort, an dem der Verkehr beiderseitig reguliert wird (vgl. Bredow 2014, S. 20). Das internationale Wirtschaftsrecht ist transnational, als eine Verschränkung von nationalem und Völkerrecht, doch besteht aufgrund der staatlichen Souveränität keine Pflicht zur Marktöffnung, es sei denn, sie wurde zuvor vertraglich vereinbart. Hier besteht ein Spannungsverhältnis zwischen dem Schutz der eigenen Volkswirtschaft als sicherer Ort des Wirtschaftens innerhalb staatlicher Grenzen, an dem der eigene Staat die Regeln bestimmt, und der Partizipation an der Weltwirtschaft, die für viele Unternehmen essenziell ist, gerade wenn es um Zugang zu ausländischen Absatzmärkten geht oder um grenzüberschreitende Arbeitsteilung z.B. im Sinne globaler Fertigungsketten (vgl. Arnauld 2019, S. 430; siehe auch Hungerland/Teupe in diesem Band). Eine lineare Entwicklung ist hier nicht zu verzeichnen, insbesondere vor dem Hintergrund eines nachhaltigen, umweltgerechten und fairen Wirtschaftens. Die Idee des fairen weltweiten Handels durch machtunabhängigen und zollreduzierten Zugang zu Märkten wird durch die protektionistische Handelspolitik der EU und zuletzt durch die unilaterale Sanktionspolitik der USA konterkariert, während gerade im Fall der USA eher auf Neuverhandlung bilateraler Abkommen gesetzt wird statt auf Einhaltung weltweit geltender Regeln für alle (vgl. Arnauld 2019, S. 435).

\section{Prozesse der Entgrenzung}

3.1 Grenzen jenseits des Staates: Überwindung oder Fortschreibung von Staatlichkeit mit Blick auf Grenzverständnisse

Der Staat und seine Staatsgrenzen sind vielfach durchlässig für Prozesse überstaatlicher Rechtsregulierung bzw. grenzüberschreitende Wahrnehmung von Aufgaben, die der Staat allein nicht zu erfüllen vermag oder besser im Zusammenspiel mit anderen erfüllen kann. Die Einbindung 
europäischer Staaten in eine supranationale Europäische Union verdeutlicht eine Entgrenzung der staatlich zentrierten Präsenz ebenso wie der Begriff der Globalisierung, der eine vergrößernde Transformation von Organisationen umschreibt, die das menschliche Zusammenleben regeln, mit der intendierten Folge, dass zwischen geografisch weit entfernten Gesellschaften Beziehungen entstehen (vgl. Held 2007, S. 12). Allerdings bedeutet dies nicht, dass es sich um einen universellen Prozess handelt, der alle gleich beteiligt, harmonisch verläuft und automatisch zur fortgesetzten Irrelevanz von Grenzen führt. Vielmehr bleibt der Staat zentraler Akteur, der Prozesse in einem Setting mitgestaltet, das dessen Machtstrukturen durch neue Akteure umgestaltet (vgl. ebd.) und letztlich so ein Handeln innerhalb und außerhalb der eigenen Grenzen ermöglicht. Staaten etablieren zuweilen flexible Interpretationsräume rund um Grenzen, auch um teils störenden Rechtspflichten zu entgehen. Die Offenheit des Grundgesetzes für internationale Zusammenarbeit zeigt, dass die Ausübung deutscher Staatsgewalt nicht im Sinne eines strengen Territorialitätsprinzips auf das deutsche Staatsgebiet beschränkt bleibt (vgl. Becker 2004a, S. 207). Doch selbst überstaatliche, also supranationale, transnationale oder internationale Zusammenschlüsse orientieren sich hinsichtlich Geltung und Wirkung ihrer Rechtsregeln im ersten Zugriff an Territorien, also Staatsgebieten der jeweiligen Mitgliedstaaten. So verbleiben bei der Eurasischen Union (EAWU) die Bereiche Staatsangehörigkeit und Grenzschutz allein im Souveränitätsbereich der Mitgliedstaaten, beziehen sich also weiter auf das begrenzte staatliche Territorium (vgl. Belozerov 2019, S. 49). Weit darüber hinausgehend schufen Zusammenschlüsse in Südamerika wie die Andengemeinschaft (CAN) und der Gemeinsame Markt im südlichen Lateinamerika (Mercosur) gemeinsame Märkte und zudem Erleichterungen der Freizügigkeit (vgl. Brumat 2020, S. 2). Doch dies bedeutet nicht ein Lossagen von der begrenzten Staatsbürgerschaft, sondern etabliert vielmehr eine funktionale Ergänzung, wie selbst auch die weitgehende Freizügigkeit der Unionsbürger*innen in der EU ausdrücklich am „Hoheitsgebiet der Mitgliedstaaten“ orientiert bleibt (Art. 21 Abs. 1 Vertrag über die Arbeitsweise der Europäischen Union, AEUV). Es gibt zwar eine EU-Außengrenze, doch die EU konstituiert kein explizit neues, eigenes EU-Territorium. Die Entscheidung darüber, wer eine staatliche Grenze überschreiten darf, gehört zu den hoheitsrechtlichen Kompetenzen, die sich Staaten nur sehr ungern nehmen lassen. Eine überstaatliche (Rechts-)Integration, wie die WTO, internationale (Schieds-)Gerichte, internationale Menschenrechtsverträge, die UN etc., bzw. regionale, wie z.B. die Europäische Union, der Mercosur oder die Westafrikanische Union, können sektoral überstaatliche Rechtsregime installieren, die staatlichen Grenzen in den spezifischen Bereichen ihre Bedeutung nicht nehmen, sondern modifizieren oder verringern. Das ist bei der Euro-Währung ebenso erkennbar wie beim sogenannten Schengen-Visum, das, einmal rechtens ausgestellt, grundsätzlich für alle EU-Mitgliedstaaten gilt. Ähnliches ist auch für die Westafrikanische Wirtschafts- und Währungsunion (UEMOA) zu verzeichnen, wo ein Binnenmarkt mit Freizügigkeit und eigener, grenzübergreifender Währung etabliert wurde. Die Idee des sogenannten Schengen-Raums ist es, die Binnengrenzen abzubauen und die EU-Außengrenzen im Gegenzug zu verstärken, wobei die Durchführung der Grenzpolitik ihrerseits mit den völkerrechtlichen Anforderungen der Genfer Flüchtlingskonvention (GFK) und dem europäischen Asyl- und Migrationsrecht in Einklang stehen müssen (vgl. Farahat/Markard 2017, S. 1088f.). Mit geografisch gebündelten Zusammenschlüssen entstehen so unterschiedliche Grenzen mit unterschiedlichen rechtlichen Bedeutungszuschreibungen.

Die Bereiche Gesundheit und Umwelt sind als rechtlich-politische Phänomene schon lange als grenzüberschreitend angesehen, doch die Ergebnisse mit Blick auf Entgrenzung sind rechtlich 
eher ambivalent. Die Nutzung der Umwelt (Land, Wasser, Luft) durch Staaten lässt sich zunächst über territoriale Zuständigkeiten vor dem Hintergrund gedeihlicher Nachbarschaft verhandeln, doch die Auswirkungen von Umweltschäden lassen sich nicht durch rechtlich-politisch gesetzte Grenzen einhegen (siehe z.B. Reaktorunfall Tschernobyl). Vielmehr steht hier dem Grundsatz der Gebietshoheit und territorialen Integrität ein deutlich sichtbarer Prozess der Entterritorialisierung entgegen (vgl. Arnauld 2019, S. 400). Rechtlich wurde schon im wegweisenden Trail-Smelter-Fall (Kanada/USA) durch ein Schiedsgericht festgestellt, dass kein Staat das Recht habe, sein Territorium in einer Art zu nutzen oder durch Unternehmen/Private nutzen zu lassen, sodass ernsthafte Schäden auf dem Territorium eines anderen Staates verursacht werden (Schiedssprüche v. 16.4.1938 und 11.3.1942, RIAA III, 1905). Bei der Durchsetzung derartiger Prinzipien bestehen jedoch Defizite. Die als Ergebnisse von Weltklimakonferenzen mehrheitlich rechtlich nicht verbindlichen Instrumente wie Codes, Standards und Leitlinien zielen als „soft law“ (Proelß 2019, S. 532) eher auf eine Form der Selbstverpflichtung der Staaten und der dort ansässigen Unternehmen. Grenzüberschreitende Kooperation in Gesundheitsfragen ist notwendig, da eine lokale Antwort auf Krankheiten, die durch globales Reisen und Wirtschaften verstärktes grenzüberschreitendes Gefährdungspotenzial haben, nicht mehr als ausreichend angesehen wird (vgl. Long 2011, S. 93ff.). Doch gerade mit Blick auf den Gesundheitsschutz ist die Entwicklung ambivalent, denn den weltweit vernetzten Forscher*innen und Firmenkooperationen stehen krisenbedingte Rückzüge auf die Grenzen eigener Staatlichkeit entgegen, die das Beispiel der grenzzentrierten Reaktionen der Staaten auf die Corona-Pandemie zeigt. Mit Blick auf das Sozialrecht und die damit verbundene Sicherung der Lebensqualität von Menschen oder deren Unterstützung in prekären Lebenssituationen bleibt zumeist der (eigene) Staat verantwortlich, so auch nach deutschem Recht (vgl. $\mathbb{S} 30$ SGB I), doch entwickeln sich selbst hier überstaatliche Zusammenhänge, die das Territorialprinzip einschränken, ersichtlich z.B. an der Rechtsprechung des Gerichtshofs der EU (vgl. EuGH C-245/94 bzgl. Erziehungsgeld von Grenzgänger*innen; EuGH C-120/95 und C- 158/96 bzgl. grenzüberschreitender Kostentragung von Brillen oder Zahnbehandlungen).

Rechtliche Herausforderungen für das raum- und grenzbezogene Denken stellen sich bezüglich der Verortung des Cyberspace und beispielsweise der Auswirkungen von (Straf-)Rechtsverstößen, die an einem Ort der Welt begangen werden, aber Auswirkungen an einem ganz anderen Ort haben und so die gängigen Muster der Zuständigkeiten von Staaten und deren Behörden bzw. Gerichten, ihren jeweils begrenzten Kompetenzradius, hinterfragen lässt (vgl. Arnauld 2019, S. 389f.). So mag z.B. die Versteigerung von Nazi-Memorabilien über das Internet an einigen Orten der Welt erlaubt und damit das Erstellen solcher Angebote möglich sein, bleibt aber in Frankreich und Deutschland verboten, von wo aus über das Internet aber eine Beteiligung an der Versteigerung möglich wäre - was abgesehen von der Strafbarkeit einzelner Personen auch zur Sperrung der Software des Versteigerungsanbieters führen kann (vgl. hierzu den Fall der Versteigerung über Yahoo und dessen Verhandlungen vor französischen und US-Gerichten: insbesondere TGI Paris, Beschluss v. 22.5.2000; dazu Arnauld 2019, S. 391). Selbst das Internet kennt eine gewisse Ortsgebundenheit, mit Blick auf Unternehmen als Anbieter, einzelne Menschen als Akteure mit Staatszugehörigkeiten sowie Staaten, die Programme sperren, Server abschalten oder Nutzerzugänge teilweise oder flächendeckend kontrollieren können. Ein letztes Schlaglicht soll auf das Völkerstrafrecht geworfen werden, denn hier vereinen sich Elemente des Völkerrechts, das die Rechtsquelle für Tatbestände der Kriegsverbrechen, der Verbrechen gegen die Menschlichkeit und gegen den Frieden ist, und 
solche des nationalen Strafrechts, weil eine individuelle Handlung einer Einzelperson unter Strafe gestellt wird (vgl. Satzger 2020, S. 322). Durch das deutsche Völkerstrafgesetzbuch wird es also vor deutschen Gerichten ermöglicht, Kriegsverbrechen, die auf anderen Territorien begangen wurden, zu verhandeln und abzuurteilen, ganz im Sinne des Weltrechtsprinzips, das den Schutz von Rechtsgütern beschreibt, deren Schutz im gemeinsamen Interesse aller Staaten ist (ebd., S. 44).

Die fortschreitende Öffnung der Staaten für überstaatliche Kooperation wird jedoch in der Krise - sei sie auf Gesundheitsschutz, Terrorismusbekämpfung oder schlicht Migration bezogen - grundlegend hinterfragt: In der Krise setzt sich der Staat, teils unter Rückgriff auf die Kategorie des Ausnahmezustandes, mit und in seinen Grenzen durch, suggeriert Stabilität, Schutz und Sicherheit, wo möglicherweise Kooperation und Solidarität wirkmächtiger wären.

\subsection{Funktionalisierung der Grenze und Externalisierung des EU-Grenzschutzes}

Eine der weltweit wohl bekanntesten Grenzen ist die zwischen Nord- und Südkorea: Hierbei geht es um die Erhaltung einer statischen Grenze, die von außen deutlich sichtbar ist. Dagegen sind beispielsweise die Seegrenzen der EU-Mitgliedstaaten im Mittelmeer zwar rechtlich bestimmbar, aber nicht sichtbar. Dabei kommt dem tatsächlichen Grenzverlauf für die Bewertung von Amtshandlungen von Grenzschutzbeamt*innen gerade mit Bezug auf Flucht elementare Bedeutung zu, denn nur wenn der Grenzverlauf eindeutig zugeordnet werden kann, können wichtige Anschlussfragen wie die nach der rechtlichen Zuständigkeit für das Entgegennehmen des Gesuchs nach Asyl und die damit zusammenhängende Verantwortlichkeit für die Behandlung des Schutzsuchenden beantwortet werden (vgl. Känner 2019, S. 17f.). Zur Koordinierung des Grenzmanagements an den Außengrenzen der EU und damit auch den See-Außengrenzen der EU-Mitgliedstaaten ist die Europäische Agentur für die Grenz- und Küstenwache Frontex (Frontières extérieures) gegründet worden, um die „Wirksamkeit einer integrierten europäischen Grenzverwaltung in der Praxis zu gewährleisten“ (Erwägungsgrund 5 FrontexVO). Dies bedeutet, dass die Kompetenzen der EU-Mitgliedstaaten institutionell koordiniert werden, wodurch die praktische Zusammenarbeit an der Grenze gewährleistet werden soll (vgl. Tohidipur 2019, S. 243). Die Kompetenzen der EU hinsichtlich der Gestaltung des Grenzschutzes, der Asylpolitik und der Einwanderungspolitik basieren maßgeblich auf Art. 77, 78 und 79 des AEUV, auf deren Basis neue Rechtsgrundlagen wie der Schengener Grenzkodex und der Visakodex, also Regeln und Verfahren für den regulären Grenzübertritt und die Kontrollmechanismen, verabschiedet wurden. Dies ist deswegen für Flüchtende existenziell, weil ein Anspruch auf Schutz erst mit Überschreiten der Grenze eines Staates gegenüber dem Staat reklamiert werden kann (vgl. Hathaway/Foster 2015, S. 288f.). Zwischen Norm und Praxis an der Grenze besteht schon seit Längerem eine große Diskrepanz. So ist es eine oft zu beobachtende Praxis, dass Schutzsuchenden der europarechtlich geforderte Zugang zu rechtstaatlichen Verfahren durch - rechtwidrige - sofortige Zurückweisung an den Land- und Seegrenzen verwehrt wird: so z.B. durch teils gewalttätige Zurückweisung ohne Prüfung des Anliegens an der kroatisch-bosnischen und ungarisch-serbischen Grenze (vgl. Känner 2019, S. 24f.) bzw. durch Abdrängen von Booten oder durch Ignorieren von Hilferufen mit Verweis auf die Zuständigkeit anderer Behörden wie etwa die sogenannte libysche Küstenwache (vgl. Tohidipur 2019, S. 248f.). Da zwischen den Mitgliedstaaten der EU schon seit Langem keine 
solidarische Einigung über den Umgang mit Migration zu erzielen ist, setzt die EU auf Externalisierung des EU-Außengrenzschutzes (vgl. Farahat/Markard 2017, S. 1096). Externalisierung des Grenzschutzes zielt in erster Linie auf politische und verwaltungstechnische Kooperation mit Nachbarstaaten der EU, wie Libyen und die Türkei, entsprechend der Strategie einer der Mitinitiatoren des sogenannten EU-Türkei-Deals: „In the end, border control depends most of all on EU's neighbours, and whether these are willing and able to stop irregular migrants from reaching the EU's borders“ (European Stability Institute 2015, S. 4). Dabei geht es vorrangig um die Ausrüstung und sicherheitstechnische Ausbildung ausländischer Dienste wie der sogenannten libyschen Küstenwache, die Fluchtbewegungen in Richtung EU unterbinden sollen (vgl. Tohidipur 2019, S. 250). Die Strategie der EU mit Blick auf die Externalisierung des Grenzschutzes fokussiert aber in der Gesamtheit einen wesentlich größeren Grenzvorraum, der insbesondere bis nach Zentral- und Westafrika reicht, wie die Frontex-Arbeitsabkommen mit Nigeria und Cap Verde und die Ergebnisse des EU-Gipfel von Valetta 2015 mit seinem Aktionsplan zeigen (EU Valetta Action Plan 2015, S. 6), wonach in Zentral- und Westafrika das Grenzmanagement verbessert werden soll. Torpediert wird dabei allerdings auch der Binnenmarkt der Westafrikanischen Wirtschaftsunion (vgl. Jakob/Schlindwein 2017, S. 131ff.). Zudem werden Fluchtbewegungen nicht verhindert, sondern die Menschen nur auf gefährlichere Wege getrieben. Der gesamte Raum um die EU herum wird so in weitem Umfang zum Grenzraum der EU.

Doch selbst die bestehenden und bestimmbaren Landgrenzen werden zunehmend ,funktional definiert bzw. als rein ,operative' Grenze bezeichnet und somit ihre Bedeutung als Umgrenzung des Schutzraums, dessen Übertreten unmittelbare Rechtswirkungen wie z.B. den Anspruch auf Durchführung eines Asylverfahrens erzeugt, minimiert. Um eine Praxis grundsätzlich rechtswidriger pushbacks zu legitimieren, wurde behauptet, dass die Zaunanlage rund um die spanische Exklave Melilla lediglich eine zusätzlich zur Staatsgrenze bestehende ,operative' Grenze sei und erst dahinter spanisches Territorium beginne, wo ein Antrag auf Asyl zu stellen sei (vgl. Känner 2019, S. 22). Und während zunächst eine Kammer des Europäischen Gerichtshofs für Menschenrechte (EGMR) diese Praxis 2017 als Verstoß gegen die Europäische Menschenrechtskonvention (EMRK) bewertet hatte, hat leider die Große Kammer des EGMR 2020 abschließend einen Rechtsverstoß verneint und somit diese Praxis quasi legitimiert (EGMR Grand Chamber, Case of N.D. and N.T. v. Spain, Applications nos. 8675/15 and 8697/15; vgl. dazu European Center for Constitutional Rights (ECCHR), Falldossier 2020). Ganz in diesem Sinne hatten auch andere EU-Mitgliedstaaten, darunter auch Deutschland, über die Möglichkeit der Einrichtung von Transiträumen rund um die bzw. an der eigenen Außengrenze diskutiert bzw. diese eingerichtet, wodurch ein Grenzübertritt schon vor der Grenze, trotz staatlicher Kontrolle über diesen Bereich, verhindert werden sollte (Stichwort: Fiktion der Nichteinreise), was rechtlich höchst problematisch ist (vgl. dazu ganz gegensätzlich Schmalz 2018; Thym 2018).

\section{Kritiken der Grenze}

\subsection{Staatsgrenzen als Grenzen der Verantwortlichkeit?}

Staaten üben ihre territoriale Souveränität zunächst begrenzt auf ihr Staatsgebiet aus. Konstituieren Staatsgrenzen damit zugleich die Grenzen eigener (staatlicher) Verantwortlichkeit? 
Zugleich führen Staaten jenseits ihres Territoriums Aufgaben aus, wie z.B. die Mitarbeit in internationalen Institutionen, die Geheimdiensttätigkeit oder die Vornahme von Kriegshandlungen auf dem Territorium anderer Staaten. Inwieweit sind Staaten an Grund- (bzw. Menschen-)Rechte gebunden, wenn die Auswirkungen ihrer Tätigkeiten lediglich Ausländer*innen betreffen, die sich außerhalb des eigentlichen Staatsterritoriums aufhalten? Die diesbezügliche Staatenpraxis und die (völker)rechtliche Beurteilung dieser Frage driften deutlich auseinander. Die deutsche Bundesregierung beispielsweise sieht hier keine Bindung durch das Grundgesetz gegeben, da dies eben nur für das deutsche Territorium gelte (vgl. Tuchtfeld 2020). Dem widersprechend hatte das Bundesverfassungsgericht schon früh festgestellt, dass deutsche Grundrechte die deutsche Staatsgewalt auch dann binden, wenn und soweit Wirkungen ihrer Betätigung im Ausland eintreten (BVerfGE 6, 290 (295); vgl. ausführlich Becker 2004b, S. 581ff.). Im Fall des von den USA eingerichteten Gefängnisses von Guantanamo im Süden Kubas und damit außerhalb des US-Territoriums bestreiten die USA die Anwendung verfassungsmäßiger Rechte aus der US-Verfassung für die Insassen des Gefängnisses (ausführlich hierzu mit Verweis auf weitere Quellen: Crawford 2019, S. 197). Dem entgegen hat der Internationale Gerichtshof (IGH) in einem anderen Fall entschieden, dass die Ausübung effektiver Kontrolle über ein Gebiet ausreiche, um eine Menschenrechtsbindung des Staates auszulösen (IGH Wall Opinion I.C.J. Reports 2004, Rn. 109ff., zu den von Israel besetzten Gebieten in Palästina). Ebenso hat auch der UN-Menschenrechtsausschuss für den Fall einer Entführung im Ausland durch Geheimdienste eines Staates eine Eröffnung des Anwendungsbereichs des Internationalen Paktes über bürgerliche und politische Rechte (IPbpR) für den entführenden Staat bejaht, da ansonsten dem Staat und seinen Beamt*innen auf fremden Territorium Handlungen erlaubt wären, die auf eigenem Territorium klare Rechtsverstöße bedeuten (Communication No. 52/1979, U.N. Doc. CCPR/C/OP/1 at 88 (1984), Rn. 12.3). Der EGMR hatte zudem in einem für die Seenotrettung bedeutsamen Urteil festgestellt, dass auch die EMRK auf Hoher See anwendbar sei, wenn der Staat die volle und exklusive Kontrolle über ein Gebiet außerhalb seines Territoriums ausübe, etwa über ein Schiff, das unter seiner Flagge fahre, und somit den Personen in diesem extraterritorialen Herrschaftsbereich die Geltung der für sie einschlägigen Menschenrechte der EMRK zu gewährleisten seien (EGMR Hirsi Jamaa and others v. Italy, Application no. 27765/09 v. 23. Februar 2012, Rn. 74ff.). Es zeigt sich also, dass aus der Sicht des Rechts die Ausübung von Hoheitsgewalt auch außerhalb des eigenen Territoriums, also jenseits der Grenze, an Verpflichtungen geknüpft ist, die auch die Beachtung von Grund- und Menschenrechten einbezieht. Die eigene territoriale Grenze begrenzt folglich nicht zugleich die eigene Verantwortlichkeit.

\subsection{Grenzen als koloniales Erbe}

Ein großer Teil der Grenzen in Westasien und auf dem afrikanischen Kontinent offenbaren noch immer sichtbare Folgen kolonialer Besitzfestschreibungen europäischer Staaten, die unabhängig von den zuvor in den Regionen vorherrschenden Strukturen gesellschaftlicher Organisation die okkupierten Gebiete untereinander aufgeteilt hatten - also Grenzziehung am Reißbrett, wodurch sich auch die vielfachen geraden Grenzverläufe der Staaten ergeben (vgl. Brunner 2013, S. 24f.). So wurden zusammengehörige ethnische Gruppen vor Ort auseinandergerissen, während zugleich konkurrierende oder gar verfeindete Gruppen plötzlich auf einem Gebiet vereinigt wurden (vgl. ebd., S. 26). Auch die lateinamerikanischen Staaten 
bestehen weitgehend in den aus der Kolonialzeit entstandenen Grenzen fort, die eroberte Verfügungsbereiche widerspiegeln (vgl. Bernecker 2005, S. 11, 15ff.). Die weitreichenden Prozesse der Dekolonialisierung in Afrika und Asien in den Jahrzehnten nach dem Zweiten Weltkrieg und schon zuvor im 19. Jahrhundert in Südamerika rückten die Grenzziehung der sich neu konstituierenden Staaten wieder in den Fokus. Die völkerrechtliche Lösung, die auch in der nachfolgenden Rechtsprechung des IGH für Streitfragen zwischen unterschiedlichen afrikanischen Staaten angewandt wurde, ist das sogenannte Uti-possidetis-Prinzip. Dieses aus dem römischen Recht stammende - und damit europäisch begründete - Prinzip betont grundsätzlich den Besitzschutz: „uti possidetis, ita possideatis“ - „wie ihr besitzt, so sollt ihr besitzen“ (Vidmar 2010, S. 323; Arnauld 2019, S. 47). Übertragen auf die Grenzfrage sieht dieser Grundsatz als Akt der Befriedung, also zur Stabilisierung von Grenzstrukturen und zur Verhinderung von Grenzkonflikten oder gar -kriegen, vor, dass die internen administrativen Grenzen und die international anerkannten Grenzen der Kolonialreiche im Augenblick der Erlangung von Unabhängigkeit durch die neuen Staaten zu deren staatlichen (international anerkannten) Grenzen werden (vgl. Ipsen 2018, S. 84; grundlegend: IGH Case concerning the Frontier Dispute [Burkina Faso vs. Mali], Urt. v. 27.6.1986, ICJ Rep. 1986, 554, 586f.). Viele afrikanische Staaten haben diesen völkergewohnheitsrechtlichen Grundsatz für ihre Grenzen anerkannt, ebenso die Afrikanische Union ganz grundsätzlich, was einerseits als friedenssichernd gelten kann, zugleich aber einen Rechtszustand fortschreibt, der seine kolonialen Ursprünge nicht abstreifen kann. Eine Auseinandersetzung mit historischer Gerechtigkeit findet nicht statt (vgl. Isensee 2018, S. 58). Zudem bedeutet das Ende der Kolonialzeit nicht zugleich das Ende der Einflussnahme vielfältiger auswärtiger, teils ehemals kolonialer Akteure, wie zum Beispiel beim antikolonialen Befreiungskampf in Angola (vgl. Bothe 1977, S. 573f.) und bei der Sezession Südsudans vom (Nord-)Sudan (vgl. Brunner 2013, S. 38). Gerade auch mit Blick auf die fehlende Mitsprache bei der Grenzziehung entwickelte sich im Zuge der Dekolonisierung die Alternative eines panafrikanischen Ansatzes, der die Abgrenzung zwischen den afrikanischen Staaten zu überwinden suchte, der allerdings nur theoretisch angedacht, aber nicht verwirklicht wurde (vgl. Nesi 2011, Rn. 4). Problematisches koloniales Erbe ist auch in Asien sichtbar, so beispielsweise in den Grenzstreitigkeiten zwischen Pakistan, Indien und Bangladesch, wo erst durch einen völkerrechtlichen Grenzvertrag 2015 Hoffnung auf Befriedung aufkam (India \& Bangladesh Land Boundary Agreement 2015; vgl. Nayar 2020). Diese Schlaglichter zeigen die problematische Fortschreibung kolonialstaatlicher Strukturen, gegründet auf einer Vorstellung berechenbarer Stabilität, die bis heute Grenzverläufe und -konflikte bestimmen.

\subsection{Wegfall von Grenzen}

Aus juristischer Perspektive könnte gefragt werden, ob es eine rechtliche Pflicht zur Grenzziehung gibt. Ist die weltweite Zentrierung auf Staatsgrenzen zwingend? Dagegen spräche, dass Menschen als Spezies schon aufgrund ihres Seins und ihrer grundsätzlich gemeinsamen Rationalität eine planetenübergreifende Gemeinsamkeit haben, die sich in kosmopolitischer Solidarität und Loyalität ausdrücken müsse, da der Zufall der Geburt an einem bestimmten Ort keine Unterscheidung des Menschen rechtfertigt und so auch die Grenzziehung als Ort der Selektion und Verhinderung der Durchwanderung und Nutzung des gesamten Planeten als ethisch untragbar erscheint (vgl. zur Diskussion Cassee 2016, S. 279; Scheffer 2019, S. 28f.). Gleichzeitig sind Staaten und damit auch Staatsgrenzen als Ordnungsmuster für Regierung und 
Verwaltung faktisch anerkannt und damit eine zunächst anzuerkennende Rechtsrealität. Doch der vermeintlich sichere ,Kokon“ staatlicher Grenzen ist auch kein Garant für Sicherheit oder gar Frieden, denn Krisen kennen keine nationalen Grenzen, ebenso wenig wie die ökologischen und menschenrechtlichen Herausforderungen in unserer Welt (vgl. Klein 2003, S. 14). Rechtlich wird hierauf mit überstaatlicher Kooperation und internationalen Verträgen reagiert, um Durchsetzung von Recht unabhängig von Grenzen zu ermöglichen und so Handlungsoptionen und Verantwortlichkeiten vom Argument des Territoriums zu entkoppeln. Zum echten Wegfall von Grenzen hat bislang aber keine überstaatliche Kooperation geführt, vielmehr werden in der Krise Grenzen reaktiviert.

\section{Fazit}

Grenzen bleiben ambivalente Konstrukte der zeitgenössischen Organisation unserer Welt. Staatsgrenzen regeln auch im Recht territoriale Markierungen zur Absicherung von Macht, an denen der Hoheitsbereich des einen Staates endet und der eines anderen beginnt (vgl. Kleinschmidt 2014, S. 3). Grenzen konturieren Territorien der Rechtsgeltung und des Schutzes, der Marktregulierung, sind aber auch - meist willkürlicher - Ausdruck kolonialer Besitzfestschreibungen, die lange Schatten werfen. Die temporäre formale Auflösung der weltweiten Blockbildung hatte nur partiell positive Konsequenzen für den Abbau von Grenzen und Mauern und trotz vielfältiger, vertiefter internationaler Integration ergaben sich neue Motive für Inund Exklusionen. Der Prozess der Globalisierung und ihn begleitende Modi supranationaler Integration wie die der EU konnten die Staatszentriertheit der Welt (noch) nicht entscheidend auflösen, Grenzen definieren weiterhin rechtlich die Aufteilung der Welt in Staaten, wobei es jedoch durch Kooperationen zu Öffnungen und erweiterten Verantwortlichkeiten auch jenseits eigener Grenzen kommt. In Bezug auf den Staat hat die Symbiose von Macht und Territorialität ihre Exklusivität verloren (vgl. Khan 2012, S. 248), wenngleich Menschen als Subjekte des Rechts noch keine nachhaltige grenzüberschreitende Anerkennung erfahren.

\section{Weiterführende Literatur}

Cassee, Andreas (2016): Globale Bewegungsfreiheit. Ein philosophisches Plädoyer für offene Grenzen. Berlin: Suhrkamp.

Farahat, Anuscheh/Markard, Nora (2017): Recht an der Grenze: Flüchtlingssteuerung und Schutzkooperation in Europa. In: Juristenzeitung (JZ) 72, H. 22, S. 1088-1097.

Isensee, Josef (2018): Grenzen. Zur Territorialität des Staates. Berlin: Duncker \& Humblot.

Pichl, Maximilian/Tohidipur, Timo (Hrsg.) (2019): An den Grenzen Europas und des Rechts. Interdisziplinäre Perspektiven auf Migration, Grenzen und Recht. Bielefeld: transcript.

Proelß, Alexander (2019): Raum und Umwelt im Völkerrecht. In: Vitzthum, Wolfgang Graf/Proelß, Alexander (Hrsg.): Völkerrecht. 8. Aufl., Berlin/Boston: De Gruyter, S. 463-584.

\section{Literaturverzeichnis}

Arendt, Hannah (1949/2011): Es gibt nur ein einziges Menschenrecht. In: Menke, Christoph/Raimondi, Francesca (Hrsg.): Die Revolution der Menschenrechte. Berlin: Suhrkamp, S. 394-410.

Arnauld, Andreas von (2019): Völkerrecht. 4. Aufl., Heidelberg: C.F. Müller.

Bauböck, Rainer (2020): Cities vs States: Should Urban Citizenship be Emancipated from Nationality? www.verfassungsblog.de/cities-vs-states-should-urban-citizenship-be-emancipated-from-nationality/, 10.9.2020.

Becker, Florian (2004a): $\$ 230$ Gebiets- und Personalhoheit des Staates. In: Isensee, Josef/Kirchhof, Paul (Hrsg.): Handbuch des Staatsrechts, Bd. XI. 3. Aufl., Heidelberg: C.F. Müller, S. 193-248.

Becker, Florian (2004b): $\mathbb{2} 240$ Grenzüberschreitende Reichweite deutscher Grundrechte. In: Isensee, Josef/Kirchhof, Paul (Hrsg.): Handbuch des Staatsrechts, Bd. XI. 3. Aufl., Heidelberg: C.F. Müller, S. 515558. 
Benhabib, Seyla (2008): Die Rechte der Anderen. Frankfurt/M.: Suhrkamp.

Bernecker, Walther L. (2005): Staatliche Grenzen - kontinentale Dynamik. Zur Relativität von Grenzen in Lateinamerika. In: Braig, Marianne/Ette, Ottmar/Ingenschay, Dieter/Maihold, Günther (Hrsg.): Grenzen der Macht - Macht der Grenzen. Lateinamerika im globalen Kontext. Frankfurt/M.: Vervuert Verlagsgesellschaft, S. 11-37.

Bothe, Michael (1977): Völkerrechtliche Aspekte des Angola-Konflikts. In: ZaöRV, Bd. 37, S. 572.

Bredow, Wilfried von (2014): Grenzen. Eine Geschichte des Zusammenlebens vom Limes bis Schengen. Darmstadt: Theiss.

Brown, Wendy (2018): Mauern. Die neue Abschottung und der Niedergang der Souveränität. Berlin: Suhrkamp.

Brumat, Leiza (2020): Personenfreizügigkeit in Südamerika. Bonn: Bundeszentrale für politische Bildung. www.bpb.de/gesellschaft/migration/laenderprofile/306418/personenfreizuegigkeit, 10.9.2020.

Brunner, Manuel (2013): „Drawing Lines upon Maps“. Ein völkerrechtlicher Beitrag zur Bedeutung von Staatsgrenzen in Afrika. In: Kettemann, Matthias C. (Hrsg.): Grenzen im Völkerrecht. Wien: Jan Sramek, S. 21-40.

Cassee, Andreas (2016): Globale Bewegungsfreiheit. Ein philosophisches Plädoyer für offene Grenzen. Berlin: Suhrkamp.

Crawford, James (2019): Brownlie's Principles of Public International Law. 9. Aufl., Oxford: Oxford University Press.

Europäischen Kommission (2015): Erklärung zur vorübergehenden Wiedereinführung von Grenzkontrollen in Deutschland, insbesondere an der deutsch-österreichischen Grenze v. 13. September 2015. https:// ec.europa.eu/commission/presscorner/detail/de/STATEMENT_15_5638, 10.9.2020.

European Center for Constitutional Rights (ECCHR) (2020): Falldossier, Fall N.D. und N.T. gegen Spanien. www.ecchr.eu/fall/nd-und-nt-gegen-spanien/\#case_case, 10.9.2020.

European Stability Institute (ESI) (2015): Why people don't need to drown in the Aegean Sea. A policy proposal, 17.9.2015. www.esiweb.org/publications/why-people-dont-need-drown-aegean-policy-propos al, 10.9.2020.

Europäische Union (2015): Valetta Summit Action Plan, 11.-12.11.2015. www.consilium.europa.eu/medi a/21839/action_plan_en.pdf, 10.9.2020.

Farahat, Anuscheh/Markard, Nora (2017): Recht an der Grenze: Flüchtlingssteuerung und Schutzkooperation in Europa. In: Juristenzeitung (JZ) 72, H. 22, S. 1088-1097.

Feichtner, Isabel (2019): Förderung des Gemeinsamen Menschheitserbes in der Tiefsee. In: Kritische Justiz (KJ) 52, H. 1, S. 10-26.

Genova, Nicholas de (2017): The Borders of „Europe“. Durham/London: Duke University Press.

Guérot, Ulrike (2019): Was ist die Nation?. Göttingen: Steidl Verlag/ifa.

Habermas, Jürgen (2015): Der Demos der Demokratie - eine Replik. In: Leviathan 43, H. 2, S. 145-154.

Hathaway, James/Foster, Michelle (2015): The Law of Refugee Status. 2. Aufl., Cambridge: Cambridge University Press.

Heintzen, Markus (1988): Auswärtige Beziehungen privater Verbände. Berlin: Duncker \& Humblot.

Held, David (2007): Mythen der Globalisierung. In: Beck, Ulrich (Hrsg.): Generation Global. Frankfurt a.M.: Suhrkamp, S. 12-26.

Hess, Sabine/Karakayali, Serhat (2017): Fluchtlinien der Migration. Grenzen als soziale Verhältnisse. In: Hess, Sabine/Kasparek, Bernd/Kron, Stefanie/Rodatz, Mathias/Schwertl, Maria/Sontowski, Simon (Hrsg.): Der lange Sommer der Migration. Grenzregime III. Berlin/Hamburg: Assoziation A, S. 25-37.

Hruschka, Constantin (2019): Binnengrenze $\neq$ Außengrenze: Klärendes vom EuGH zur Wiedereinführung von Grenzkontrollen. www.verfassungsblog.de/binnengrenze- \%E2\% 89\%A0-aussengrenze-klaerendes-v om-eugh-zur-wiedereinfuehrung-von-grenzkontrollen, 10.9.2020.

Ipsen, Knut (2019): Völkerrecht. 7.Aufl., München: C.H. Beck.

Isensee, Josef (2018): Grenzen. Zur Territorialität des Staates. Berlin: Duncker \& Humblot.

Jakob, Christian/Schlindwein, Simone (2017): Diktatoren als Türsteher Europas. Wie die EU ihre Grenzen nach Afrika verlagert. Berlin: Ch. Links.

Jellinek, Georg (1960): Allgemeine Staatslehre. 3. Aufl., Nachdruck, Darmstadt: Wissenschaftliche Buchgesellschaft.

Jennings, R.Y. (1963): The Acquisition of Territory in International Law. Manchester: Manchester University Press.

Känner, Lise (2019): Zur rhetorischen Verschiebung von Staatsgrenzen. In: Pichl, Maximilian/Tohidipur, Timo (Hg.): An den Grenzen Europas und des Rechts. Interdisziplinäre Perspektiven auf Migration, Grenzen und Recht. Bielefeld: transcript, S. 17-40.

Khan, Daniel-Erasmus (2019): Grenze, II. Rechtlich, Version 22.10.2019. In: Staatslexikon, 8. Aufl. www.staatslexikon-online.de/Lexikon/Grenze, 10.9.2020. 
Khan, Daniel-Erasmus (2012): Territory and Boundaries. In: Fassbender, Bardo/Peters, Anne (Hrsg.): The Oxford Handbook of The History of International Law. Oxford: Oxford University Press, S. 225-249.

Khan, Daniel-Erasmus (2004): Die deutschen Staatsgrenzen: rechtshistorische Grundlagen und offene Rechtsfragen. Tübingen: Mohr Siebeck.

Klein, Eckart (1980): Statusverträge im Völkerrecht. Berlin/Heidelberg/New York: Springer.

Klein, Naomi (2003): Über Zäune und Mauern. Berichte von der Globalisierungsfront. Frankfurt/M.: Büchergilde Gutenberg.

Kleinschmidt, Christoph (2014): Semantik der Grenze. In: APuZ 4-5/2014, S. 3-8.

Long, William J. (2011): Cross-border health cooperation in complicated regions: The case oft the Mekong Basin Disease Surveillance Network. In: Cheema, G. Shabbir/McNally, Christopher A./Popovski, Vesselin (Hrsg.): Cross-Border Governance in Asia. Regional Issues and Mechanisms. New York: UN University Press, S. 93-121.

Marx, Reinhard (2020): Aufenthalts-, Asyl- und Flüchtlingsrecht. Baden-Baden: Nomos.

Nayar, Nitika (2020): India and Bangladesh: Exchanging border enclaves \& (re-)connecting with new citizens, Sambandh Scholars Speak Blog, 12.5.2020. www.brookings.edu/blog/up-front/2020/05/12/s ambandh-blog-india-and-bangladesh-exchanging-border-enclaves-re-connecting-with-new-citizens/, 10.9.2020.

Nesi, Giuseppe (2011): Uti possidetis Doctrine. In: Wolfrum, Rüdiger (Hrsg.): Max Planck Encyclopedia of Public International Law. Heidelberg: Oxford University Press.

Özdemir, Özgen (2020): Deutschland als amicus curiae. Zur Debatte um die Staatlichkeit Palästinas als Voraussetzung der Jurisdiktion des Internationalen Strafgerichtshofs. https://voelkerrechtsblog.org/articl es/deutschland-als-amicus-curiae, 10.9.2020.

Proelß, Alexander (2019): Raum und Umwelt im Völkerrecht In: Vitzthum, Wolfgang Graf/Proelß, Alexander (Hrsg.): Völkerrecht. 8. Aufl., Berlin/Boston: De Gruyter, S. 463-584.

Satzger, Helmut (2020): Internationales und Europäisches Strafrecht. 9. Aufl., Baden-Baden: Nomos.

Scheffer, Paul (2019): Wozu Grenzen? Freiheit in Zeiten von Globalisierung und Migration. München: Carl Hanser.

Schliesky, Utz (2004): Souveränität und Legitimität von Herrschaftsgewalt. Tübingen: Mohr Siebeck.

Schmalz, Dana (2018): Die Fiktion der Nichteinreise ist ein Instrument der Entrechtung. www.verfassungs blog.de/die-fiktion-der-nichteinreise-ist-ein-instrument-der-entrechtung/, 10.9.2020.

Schöbener, Burkhard/Knauff, Matthias (2019): Allgemeine Staatslehre. München: C.H. Beck.

Schorkopf, Frank (2017): Staatsrecht der internationalen Beziehungen. München: C.H. Beck.

Schulze, Hagen (1990): Die Wiederkehr Europas. Berlin: Corso bei Siedler Verlag.

Shah, Anjasi/Tripathi, Ankitashri (2020): The citizenship test in India. www.voelkerrechtsblog.org/the-citi zenship-test-in-india/, 10.9.2020.

Thym, Daniel (2018): Für ein „Helsinki“ im deutschen Migrationsrechtsdiskurs. www.verfassungsblog.de/ fuer-ein-helsinki-im-deutschen-migrationsrechtsdiskurs/, 10.9.2020.

Tohidipur, Timo (2019): Die Rolle der Menschenrechte in der Arbeit von Frontex. In: Kugelmann, Dieter (Hrsg.): Polizei und Menschenrechte, Bonn: Bundeszentrale für politische Bildung S. 241-258.

Tohidipur, Timo (2015): Formalisierte Staatsverständnisse als Gegenstand des Verfassungstransfers. In: KritV 98, H. 2, S. 108-129.

Tohidipur, Timo (2011): Justiz als Wächterin der Menschenrechte: Gerichtshöfe in Europa und ihr Verhältnis zur politisch-demokratischen Konstitution. In: Heinrich Böll Stiftung (Hrsg.): Dossier: Grenzstatt Menschenschutz? https://heimatkunde.boell.de/sites/default/files/dossier_asyl-_und_fluechtlingspoli tik.pdf, 10.9.2020.

Triepel, Heinrich (1939/1940): Die auswärtige Politik der Privatperson. In: ZaöRV, Bd. 9, S. 1-30.

Tuchtfeld, Erik (2020): Grundrechte schützen - eine grenzenlose Verpflichtung?. www.voelkerrechtsblog.o rg/articles/grundrechte-schutzen-eine-grenzenlose-verpflichtung/, 10.9.2020.

Vallet, Elisabeth/David, Charles-Philippe (2017): Walls of Money: Securitization of Border Discourse and Militarization of Markets. In: Vallet, Elisabeth (Hrsg.): Borders, Fences and Walls. State of Insecurity? London/New York: Routledge, S. 143-156.

Verdross, Alfred/Simma, Bruno (1984): Universelles Völkerrecht. Theorie und Praxis. 3. Aufl., Berlin: Duncker \& Humblot.

Vidmar, Jure (2010): Confining New International Borders in the Practice of Post-1990 State Creations. In: ZaöRV, Bd. 70, S. 319-356.

Vitzthum, Wolfgang Graf (2004): \18 Staatsgebiet. In: Isensee, Josef/Kirchhof, Paul (Hrsg.): Handbuch des Staatsrechts, Band II. 3. Aufl., Heidelberg: C.F. Müller, S. 163-192.

Wolter, Detlev (2002): Völkerrechtliche Grundlagen „Gemeinsamer Sicherheit“ im Weltraum. In: ZaöRV, Bd. 62, S. 941-992. 